\title{
Angle metrology at INRIM in the framework of the EMRP Project SIB58
}

\author{
Astrua M, Pisani M and Santiano M \\ INRiM, Torino, strada delle cacce 91, Italy
}

\begin{abstract}
Résumé. Dans le cadre d'un projet européen, visant à l'amélioration de la traçabilité des mesures d'angle à l'unité radian SI, l'Institut Italien de Métrologie (INRIM) mettra au point trois nouveaux appareils. Un étalon d'angle utilisée pour étalonner artefacts d'angle, encodeurs angulaires et autocollimateurs angulaires (AC); un petit générateur d'angle utilisé pour étalonner les niveaux électroniques et les $\mathrm{AC}$ et un interféromètre de référence utilisé pour mesurer de petites inclinaisons. La précision et la résolution cible des trois dispositifs est de 1'ordre de 0,01" (50 nrad). Le principe de fonctionnement des trois dispositifs et les résultats préliminaires sont présentés.
\end{abstract}

\section{Introduction}

The measurement of angles at the uncertainty level of tens of nanoradian are extremely challenging and interesting for many applications in the field of mechanical industry, optical surface characterization or geodetic instrumentation. The best instruments to measure large angles and small angles are respectively angular encoders and autocollimators. Both instruments are extensively used in industrial, science and metrology fields, and exhibit exceptional resolutions to the level of 0.001 " or few nanoradians. Nevertheless the traceability to the angle unit, i.e. the reliability of the instrument readings is not guaranteed at the same level. Indeed today only in exceptionally complex and accurate facilities operating in few National Metrology Institutes in the world can provide angle measurements/calibrations with nanoradian level accuracy $[1,2]$. The European project "Angles" [3] is devoted to develop instruments methods and guidelines to improve the accuracy of angle measurements in various application field. In the framework of this project INRIM is working on the realization of three novel devices. The first is a special rotating table equipped with a rotating encoder, the second is a generator of very small angles based on a piezo actuator and the third is an interferometer to measure very small angles with traceability to the SI.

\section{The rotating encoder standard (RES)}

The rotating encoder standard (RES) is designed to generate and measure angles over the full circle interval with a target accuracy of 0.01 ". The device will be used to calibrate optical polygons, angular encoders and autocollimators.

\subsection{Working principle}

The measurement principle was pioneered by E. W. Palmer in 1984 [4] and it is based on a continuously rotating angular encoder, read by two heads, one fixed with respect to the laboratory frame and a second one rotating with the measurement table. Thus, the angle measurement is obtained by the measurements of the phase difference between the fixed head signal (used as a reference) and the rotating head one. As a consequence, the encoder errors are cancelled by the average made each complete revolution of the encoder. A schematic of the rotating encoder principle is reported in figure 1 .

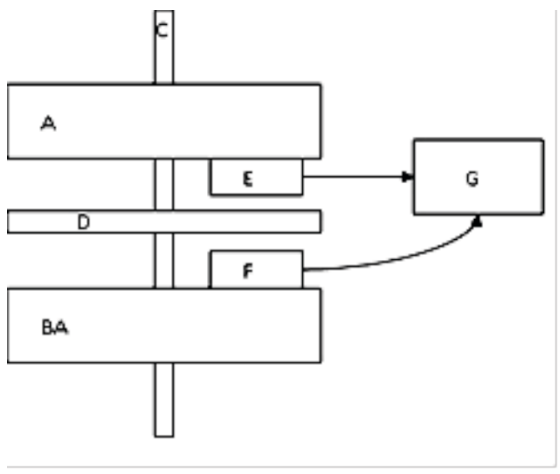

Figure 1. Schematic of the rotating encoder principle. D continuously rotating optical encoder; F: reading head fixed to the reference frame BA; E: reading head fixed to the measuring table A; G: phase measurement; $\mathrm{C}$ common rotation axis.

\footnotetext{
a Corresponding author: m.pisani@inrim.it
} 


\subsection{Realization}

The practical device is composed by three main parts: a base, an inner rotating part holding the encoders and an upper table available for the user.

The three are free to rotate reciprocally by means of air bearings. The encoder is rotated by a brushless motor integrated in the base. The encoder itself is in fact a pair of stacked encoders (Heidenhain ring encoder, model ERA 4200, 40000 lines with $20 \mu \mathrm{m}$ spacing). Two heads are faced to each ring about $180^{\circ}$ one respect to the other, for a total of four reading heads.

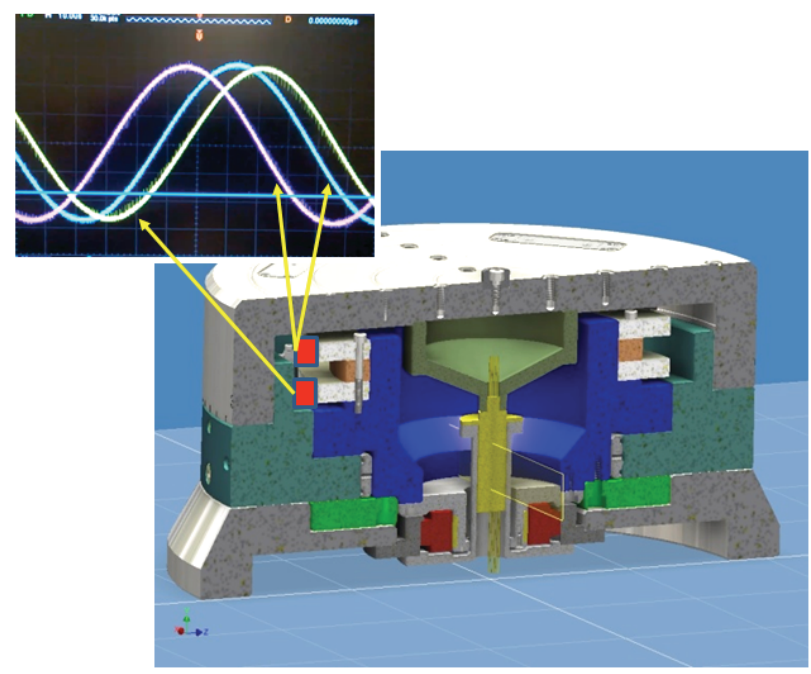

Figure 2. Section of the project of the INRIM RES and picture of the signals from the reading heads.

In figure 2, a section of the RES and picture of the signals coming from the reading heads are reported. The basement (lower gray part) supports the fixed part of the air bearing (dark green). The blue part of the air bearing, driven by the motor (red), rotates continuously. Two identical encoders (light grey) are fixed to it. Two reading heads are fixed to the base, other two are fixed to the upper part (grey) free to rotate with respect to the main structure. The signals of the moving heads are brought towards the fixed part through a sliding contact (yellow). Each head generates two quadrature sinusoidal signals that are amplified by means of an electronic circuit and sent to an analog to digital converter board (ADC, NIUSB-6259 BNC).

A Labview $®$ based program elaborates the signals captured by the ADC board and measures the phase difference between the signals coming from the two rotating heads and from one of the fixed head according to the following procedure. The reference signal is mixed with the signals of the measurement heads. The results are low pass filtered to eliminate the $20 \mathrm{kHz}$ harmonic and to deliver the average value of the product. Each complete phase revolution corresponds to a shift of one encoder line (32.4 arcsec). A Matlab ${ }^{\circledR}$ based algorithm calculates the instantaneous phase angle. The phase is then averaged over the entire revolution of the encoder. A counting logic measures the integer part of the phase (the number of complete phase revolutions) and adds or subtract the integer to the fractional measurement. The final value is converted in arcseconds and the result is stored. Preliminary tests and details about the software are described in [5].

A picture of the device, ready for a calibration of an optical polygon, is reported in figure 3 .

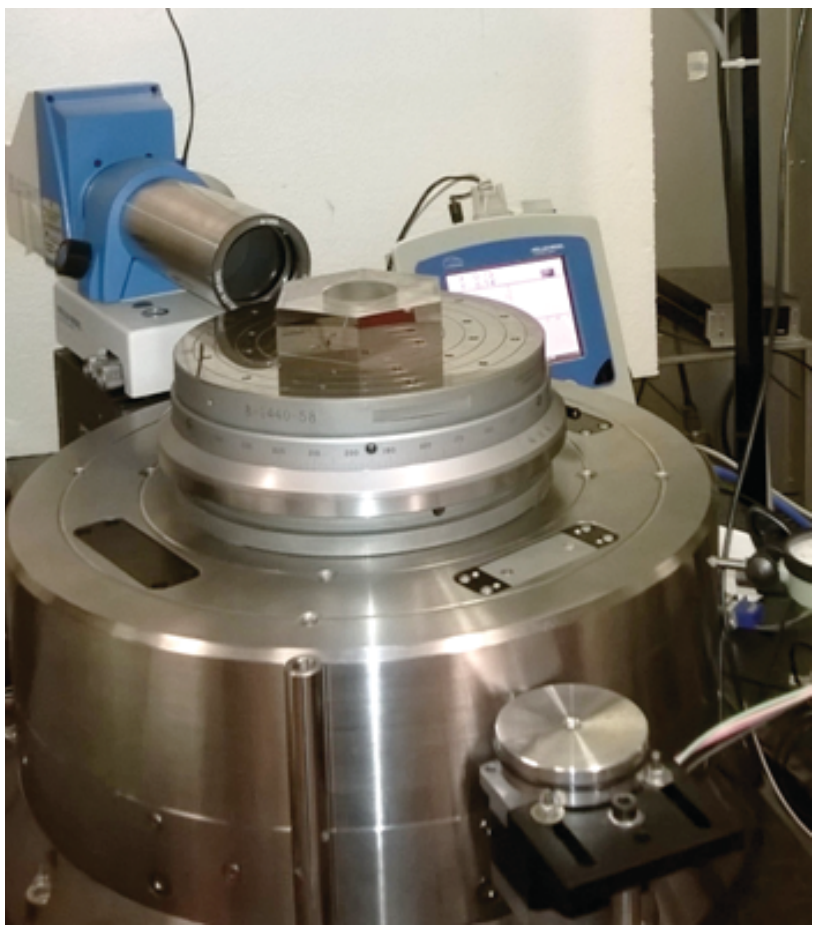

Figure 3. Picture of the RES while calibrating a polygon. An index table is used to implement the multistep technique. In the foreground is visible the driving microstep motor.

In order to automate the system, the table can be rotated by means of a microstep motor, which is visible in figure 3 bottom right.

\subsection{Preliminary results}

In order to test the system, a first autocollimator (AC) calibration and a 6-sided polygon calibration have been performed.

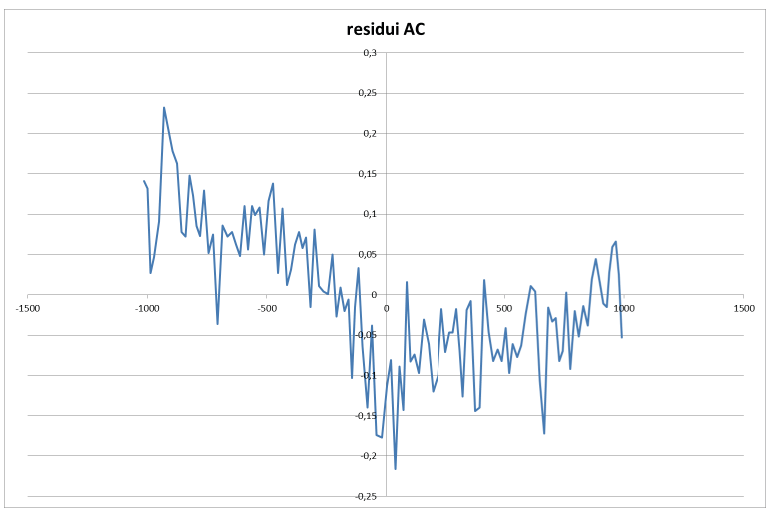

Figure 4. First calibration of an $\mathrm{AC}$ showing the high resolution of the RES. 
Moreover, a Labview program to automatically perform the calibration of optical polygons has been implemented. The software acts on the motor reading the encoder position and the autocollimator output. The software allowed to perform a first calibration of a 6-sided optical polygon with a semi-automatic procedure. Taking advantage of the multistep technique, it is possible to separate the errors of the polygon from the residual errors of the RES, which were found to be of the order of \pm 0.03 ".

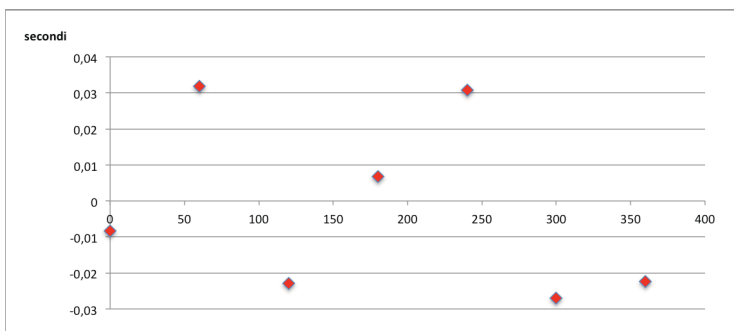

Figure 5. Residual errors of the RES as a product of a calibration of a 6 .sided polygon showing the intrinsic high accuracy of the RES.

Both measurements show a good behaviour of the device, the driving system and the software. Next work will be dedicated to the metrological validation of the instrument through comparison with other NMI's standards in the framework of the EMRP project in particular by using the shearing method described in [6].

\section{The nano angle generator (NAG)}

The nano angle generator (NAG) is designed to generate vertical tilts on a range of 2000" based on the sinebar principle (trigonometric realization of the unit radian). The instrument will be used as is or in combination with the Fizeau Interferometer (see next chapter) for improved accuracy, for the calibration of autocollimators and of electronic levels.

\subsection{Working principle and design}

The NAG will be realized from a monolithic grade 5 titanium block. The design is mainly based on the experience made at INRIM with the previous nano angle generator described in [7]. In that device we found out that the main sources of instability came out from the complex realization of the device and the miscellanea of materials used. The choice of the monolithic titanium should guarantee higher stability, higher stiffness and relatively low weight of the device. In figure 6 a rendering of the NAG is shown. The NAG is based on a stiff tilting plane connected to the reference base through elastic hinges made with wire electro-erosion. The movement is given by a piezo actuator (PI N-111.2A) equipped with a linear encoder having $25 \mathrm{pm}$ open loop resolution and $5 \mathrm{~nm}$ closed loop resolution.

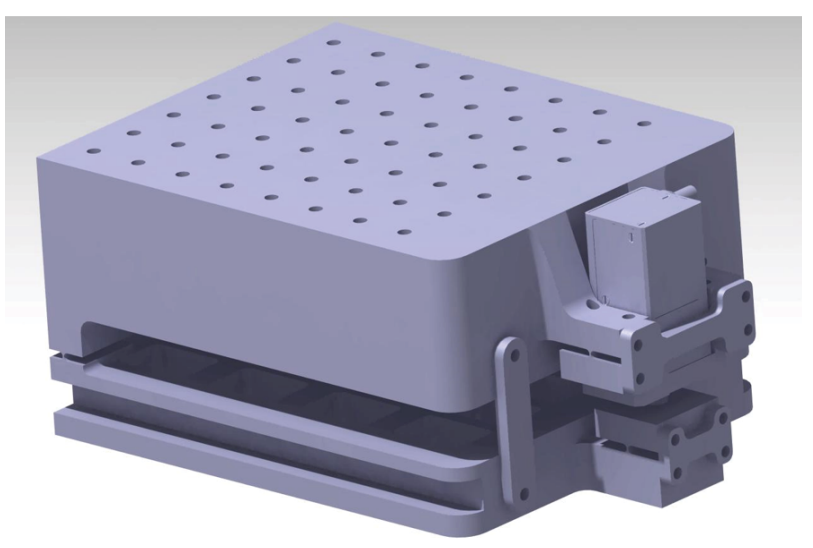

Figure 6. Drawing of the NAG made of a monolithic block of titanium and equipped with a precision piezo actuator.

The behaviour of the elastic hinges has been simulated by means of finite element analysis to find the compromise between roughness and sensitivity (fig 7). The upper plate will be used to host electronic levels and accelerometers, and the back side will be used to fix a mirror to calibrate ACs and to made the NAG to operate in combination of the FAI described in the next chapter.
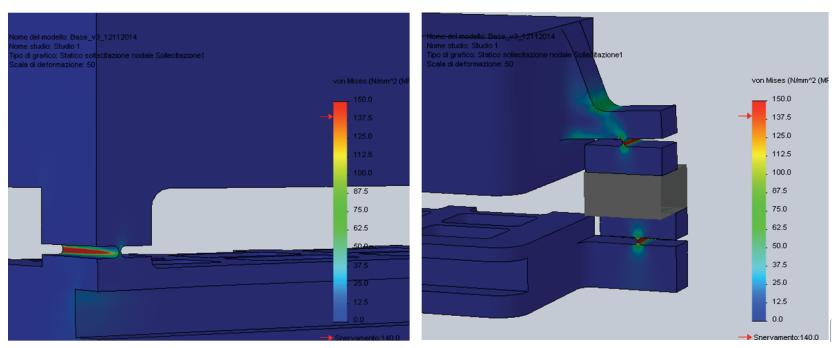

Figure 7. A detail of the finite element analysis used for the sizing of the helastic hinges.

\section{The Fizeau angle interferometer (FAl)}

The Fizeau angle interferometer (FAI) is designed to measure small tilts (vertical and horizontal) on a range of 2000 " and an accuracy target of 0.01 ". The instrument will make use of a laser wavelength for the traceability to the SI units and will be used as an extremely accurate autocollimator.

\subsection{Optical set up}

In figure 8 is represented the schematic diagram of the FAI. The Fizeau interferometer will generate interference fringes between the two reflecting flat surfaces: the reference mirror and the tilting mirror. The interferometer is fed with a collimated laser beam from a stabilized He$\mathrm{Ne}$ laser. The interference fringes are imaged on a CCD detector. The diameter of the laser beam is about $50 \mathrm{~mm}$, and the sensor surface is a square with $35 \mathrm{~mm}$ side. The structure will be mounted on a compact and stable bench. Unlike classical Fizeau interferometer used to analyse the 
quality of optical surfaces, no optical elements are interposed between the interferometer and the CCD sensor, furthermore the laser beam is made of plane wavefronts, so that the pitch of the fringes on the sensor is the same as at the interferometer level. That means that we can measure the fringe pitch in pixel units, convert it in micrometers (after proper calibration of the pixel pitch) and eventually estimate the angle between the two surfaces.

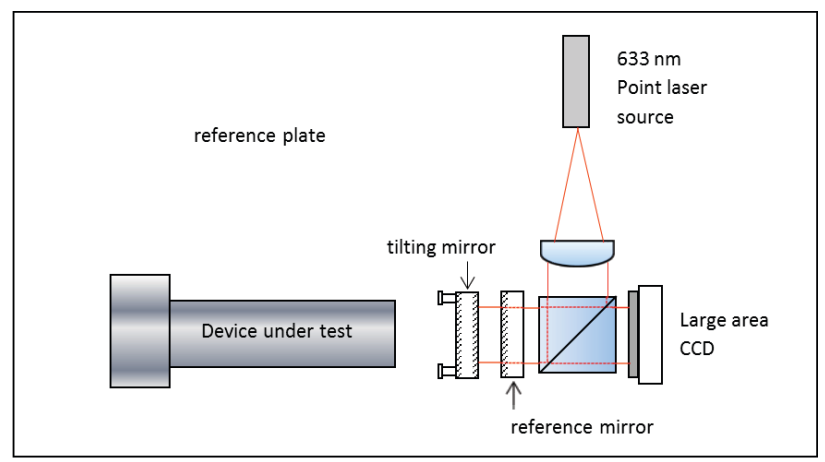

Top view

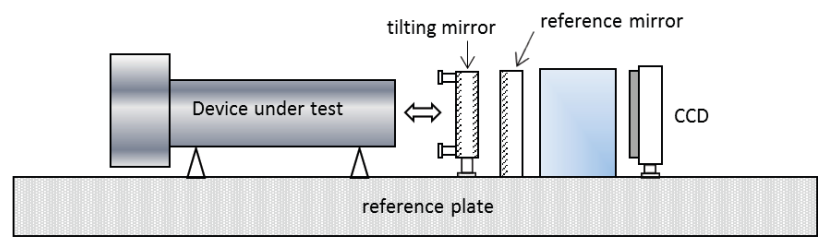

Side view

Figure 8. Schematic of the FAI. A parallel laser beam is sent to the Fizeau interferometer made of a semi-reflective reference mirror and a mirror which tilt will be measured. The interference pattern is than sent to a calibrated CCD matrix. The ratio between the laser half wavelength and the fringe pitch gives the angle in radian unit.

\subsection{Fringe analysis}

A preliminary experiment has been set-up to test the CCD and the fringe analysis software. The fringes are acquired by the CCD sensor, converted in a $2 \mathrm{D}$ matrix and elaborated by means of a Matlab based software. The images are fitted with a cosine profile which amplitude is modulated with a bidimensional Gaussian profile. The first is the theoretical profile of the interference fringes, the second is the power profile of the laser beam. In the practical final realization only the central part of the laser beam will be used so to minimize the amplitude modulation. In figure 9 a profile where the amplitude is strongly modulated has been acquired and fitted to demonstrate the correct behaviour of the fitting algorithm. In the following figures, some examples of recorded fringes and the respective fitting function for different angles are reported.
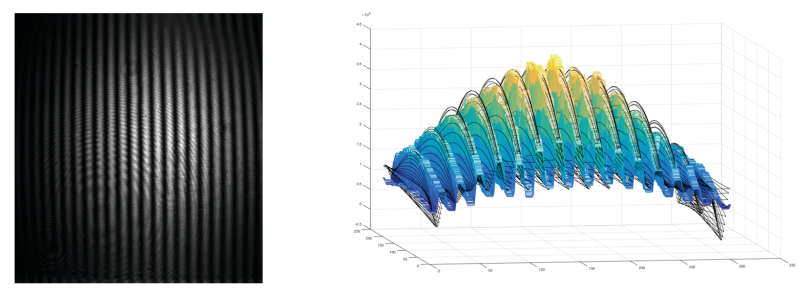

Figure 9. Left: intensity matrix imaged on the CCD sensor. Right 3D view of the fringe profile (colour surface) and the artificial function produced by the fitting algorithm (black lines).
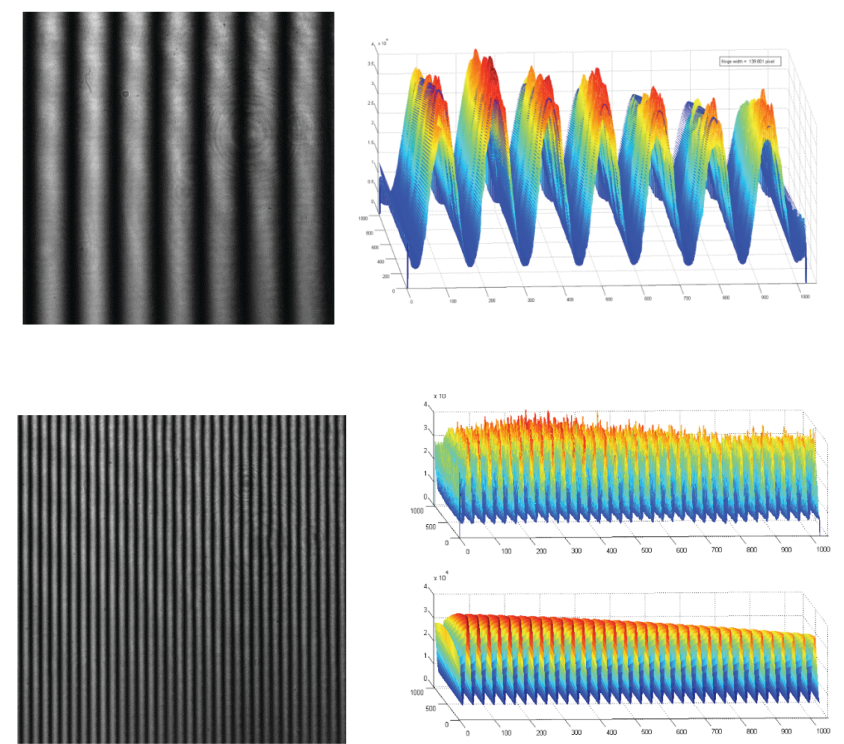

Figure 10. Interference pattern and fitting function for different angles between the two surfaces: respectively $150 \mu \mathrm{rad}$ and 710 $\mu \mathrm{rad}$.

\section{Conclusions}

Three novel devices for large and small angle measurements with target accuracy of 0.01 ” (50 nrad), have been designed and partially built and tested at INRIM. The rotating encoder standard (RES) has been already built and successfully tested and is currently under metrological validation. The nano angle generator (NAG) has been designed and is currently under construction. The Fizeau angle interferometer (FAI) has been tested for the acquisition system and software; the optical system is under construction. In the framework of the EMRP Project SIB58 the three instruments will be completed, tested and validated by comparison with the instruments of the Project's partners. 


\section{Acknowledgments}

The authors would like to acknowledge funding from EMRP SIB58 "Angles" of the European Metrology Research Programme (EMRP). The EMRP is jointly funded by the EMRP participating countries within EURAMET and the European Union.

Authors would like to thank Marco Zangirolami for his help in designing the NAG.

\section{References}

1. R Probst, R Wittekopf, M Krause, H Dangschat and A Ernst, (1998) Meas. Sci. Technol. 91059

2. Watanabe T, Fujimoto H and Masuda T (2005) Selfcalibratable rotary encoder J. Phys.: Conf. Ser. 13 240-45

3. http://www.anglemetrology.com/

4. E.W. Palmer, Precision Engineering 635988 900335 (1984)

5. M. Pisani, M. Astrua Proc. of the 13th Euspen International Conference, Berlin, (2013)

6. Ralf D Geckeler and Andreas Just (2014) Meas. Sci. Technol. 25105009

7. M. Astrua, M. Pisani, Metrologia 46 (2009) 